P637

FIRST IRISH EXPERIENCE OF HUMAN BREAST MILKBASED FORTIFIER USE IN NICU: A THEMATIC QUALITATIVE STUDY OF NURSING AND MIDWIFERY PERSPECTIVE

\begin{abstract}
${ }^{1,2}$ Roy K Philip*, ${ }^{2}$ Margo Dunworth, ${ }^{2}$ Niazy Al-Assaf, ${ }^{2}$ Deirdre O'Connell, ${ }^{2}$ Irene Bierne, ${ }^{2}$ Bernadette Murphy. 'Graduate Entry Medical School, University of Limerick, Limerick, Ireland; ${ }^{2}$ University Maternity Hospital Limerick, Limerick, Ireland
\end{abstract}

\subsection{6/archdischild-2019-epa.968}

Background Necrotising enterocolitis (NEC) is the commonest gastrointestinal emergency among premature infants, and accounts for significant morbidity and mortality. Breast milk $(\mathrm{BM})$ is considered as the best nutritional option to prevent NEC. Traditionally cow's milk-based fortifier is added to BM to optimise growth of extremely premature infants, thus potentially increasing the risk of NEC. More recently the value of replacing the traditional cow's milk-based fortifier with human breast milk-based fortifier [resulting in the concept of an exclusive human milk (EHM) based diet] has been demonstrated to reduce NEC, improve feeding tolerance, reduce dependence on parenteral nutrition and enhance growth. We report the nursing/midwifery experience of introducing EHM for the first time in Ireland, through a qualitative study.

Aims To explore nurses' and midwives' perspectives on the provision of human breast milk-based fortifier in an Irish neonatal unit for the first time.

Methods Seven senior neonatal nurse/midwives, who were involved in hands-on provision of human breast-milk based fortifier in the NICU at University Maternity Hospital Limerick (UMHL), were offered a semi-structured questionnaire. Following audit committee approval, a thematic interpretive qualitative study was conducted.

Results Four themes were identified based on 54 feeding episodes noted in 2017 and 2018 from the two extremely low birth weight (ELBW) infants who were offered human breast milk-based fortifier for the first time in Ireland. Key observations, experiences, expectations and barriers highlighted were analysed. Themes noted were, 1. breastfeeding improvement and culture, 2. feeding tolerance, 3. educational needs and support, and 4. confidence and concerns of nurses/midwives. Sub-themes identified were further evaluated.

Conclusion Supportive environment and targeted staff education within NICU would assist in maintaining high breastfeeding rates as well as implementation of an exclusive human milk diet for ELBW infants.

\section{P638 CULTURALLY SENSITIVE NEONATAL CARE PROVISION TO INFANTS OF PARENTS FROM THE TRAVELLER COMMUNITY: A NURSING AND MIDWIFERY PERSPECTIVE}

\footnotetext{
1,2Irene Beirne* ${ }^{2}$ Carmel Bradshaw, ${ }^{1,2}$ Roy Philip. 'University Maternity Hospital, Limerick,
} Ireland; ${ }^{2}$ University of Limerick, Limerick, Ireland

\subsection{6/archdischild-2019-epa.969}

Background Parents struggle to deal with fears of infant wellbeing in unfamiliar environments such as the neonatal intensive care unit (NICU). These challenges are further heightened for parents who do not perceive themselves to be fully integrated into the society in which they are receiving care, e.g. the Traveller community. Disadvantages such as poor education, socioeconomic deprivation, unemployment and poverty are more prevalent in this population. Their culture, ethnicity and nomadic lifestyle makes them often untrusting of institutions and lends itself to poor attendance, engagement and compliance with health services. For nurses/midwives to practice neonatal care with cultural sensitivity, understanding of their cultural beliefs, patients' perceptions of illness, and past experiences with healthcare providers would be important. There is paucity of focused studies on NICU staff perspectives on specific cultural issues affecting newborn infants of the Traveller community.

Aims To explore nurses' and midwives' perspectives on the provision of culturally sensitive care in a neonatal setting to infants born to parents from Traveller community.

Methods Following ethical approval, a descriptive qualitative approach was used to conduct face-to-face interviews with ten nurses/midwives from an NICU in the Mid-West of Ireland and four themes were identified.

Results Four themes noted were, 1. Barriers to breastfeeding for women from the Traveller community included sub-themes of cultural influences, impact of the Beutler test and nurse/ midwife assumptions. 2. Cultural Issues around trust, religion, rigidity of the healthcare system and social supports. 3. Educational deficits relating to poor literacy of the Traveller community resulting in barriers to seek health promotion opportunities. 4. Nurses/midwives' concerns, incorporating infant discharge, post-discharge safety and perceived exposure to domestic violence.

Conclusion Information on the nature and extent of the challenges encountered by staff in providing culturally sensitive care to the Traveller population within a neonatal setting would assist in developing strategies to enhance culturally sensitive care plans for this sub-population. Gaining trust in relation to breastfeeding and culturally held beliefs would assist nurses/midwives to offer family-centred care to the neonatal population of Traveller background. Discharge planning and follow-up needs to be tailor made to suit their geographical mobility within Ireland but also across Europe.

\section{P639 MANAGEMENT OF CONGENITAL CHYLOTHORAX IN INTENSIVE NEONATAL CARE UNIT IN SFAX (TUNISIA)}

Amel Ben Hmed, Afef Ben Thabet, Chiraz Regaieg*, Manel Charfi, Amira Bouraoui, Nedia Hmida, Ridha Regaieg, Amira Bouraoui, Abdellatif Gargouri. Department of Neonatology, Hedi Chaker Hospital, Sfax, Tunisia

\subsection{6/archdischild-2019-epa.970}

Background Congenital chylothorax (cc) is a rare condition. It consists on an accumulation of the chyle in the pleural cavity. It is associated with significant morbidities including respiratory distress, malnutrition, immunodeficiency and infections. The treatment of congenital chylothorax is still not well codified. We have focused on postnatal management and after review of the literature we propose an algorithm of the treatment of congenital chylothorax.

Methods We report five cases of congenital chylothorax admitted to our neonatal intensive care unit between 2010 and 2018. 